\title{
CATEgory Trees - Classifiers THAT BRANCH ON CATEGORY
}

\author{
Kieran Greer \\ Distributed Computing Systems, Belfast, UK
}

\begin{abstract}
This paper presents a batch classifier that splits a dataset into tree branches depending on the category type. It has been improved from the earlier version and fixed a mistake in the earlier paper. Two important changes have been made. The first is to represent each category with a separate classifier. Each classifier then classifies its own subset of data rows, using batch input values to create the centroid and also represent the category itself. If the classifier contains data from more than one category however, it needs to create new classifiers for the incorrect data. The second change therefore is to allow the classifier to branch to new layers when there is a split in the data, and create new classifiers there for the data rows that are incorrectly classified. Each layer can therefore branch like a tree - not for distinguishing features, but for distinguishing categories. The paper then suggests a further innovation, which is to represent some data columns with fixed value ranges, or bands. When considering features, it is shown that some of the data can be classified directly through fixed value ranges, while the rest must be classified using a classifier technique and the idea allows the paper to discuss a biological analogy with neurons and neuron links. Tests show that the method can successfully classify a diverse set of benchmark datasets to better than the state-of-the-art.
\end{abstract}

\section{KEYWORDS}

Classifier, tree, batch, category, centroid, neural.

\section{INTRODUCTION}

This paper presents a batch classifier that splits a dataset into tree branches depending on the category type. It has been improved from the earlier version and fixed a mistake in the earlier paper. Two important changes have been made. The first is to represent each category with a separate classifier. Each classifier then classifies its own subset of data rows, using batch input values to create the centroid and also represent the category itself. This means that for each category, a data column will try to map to a single distinct value and it is the summed difference between those column mappings that determines the output error. If the classifier contains data from more than one category however, it needs to create new classifiers for the incorrect data. The second change therefore is to allow the classifier to branch to new layers when there is a split in the data, and create new classifiers there for the data rows that are incorrectly classified. Each layer can therefore branch like a tree - not for distinguishing features, but for distinguishing categories. This is different to traditional tree solutions that typically branch on some feature value and so the new classifier is being called Category Trees.

The paper then suggests a further innovation, which is to represent some data columns with fixed value ranges, or bands. When considering features, it is shown that some of the data can be classified directly through fixed value ranges, while the rest must be classified using a classifier technique. An earlier version added branches [17] to an oscillating error technique [18]. That technique allowed the error update to be independent for each data column, meaning that it could 
oscillate around the desired output, independent of the other columns. Because that classifier worked off averaged values, it may be the case that some data can be classified directly, without weight adjustments. The averaged value is simply a single value for a whole range of inputs and so maybe a value band can represent that range as a fixed set of boundaries. This is still a secondary consideration, but it may also be possible to construct these fixed boundaries for single dimensions, to represent individual features. This determination is pre-classifier if you like and would remove categories that can therefore be recognised from distinct features. After categories and related data rows are removed using fixed data bands, the rest of the data can be used to train the classifier. For example, when using fixed bands for classification, if the input data row does not fall into one of the bands, it can be run through the classifier instead. Tests show that the method can successfully classify a diverse set of benchmark datasets to better than the state-ofthe-art.

The rest of this paper is organised as follows: section 2 introduces some related work. Section 3 reviews the earlier work on the classifier, while sections 4 and 5 describe the two improvements. Section 6 describes the possibility of using fixed bands and section 7 re-runs the test set to verify the classifier's accuracy. Finally, section 8 gives a discussion and some conclusions to the work.

\section{RELATED WORK}

This research is based specifically on two earlier papers that introduced an oscillating error technique [17][18]. The idea of using batch values came from the idea of using the input shape over distinct point values [20]. The input would produce a best fit wave shape between its points, but then it was discovered that the order of the rows could be changed, thereby changing the shape and the averaged shape value would remain the same. It was also decided that the averaged wave shape and the average data point values were essentially the same and so batch data point values were preferred instead. The earlier classifier was used to classify categorical data, or data rows grouped into categories. It used averaged values for each category and an oscillating error technique that decided whether to add or subtract the error from each cell value, to minimise the total error. The first paper [18] actually had a mistake in its evaluation, that was corrected by the branching mechanism suggested in [17]. The paper [17] is now replaced by this one, which has extended the work further. The idea of batch processing or averaged values is not new and has been used in some of the earlier neural network models, for example [15][23]. The research of this paper also considers classifying each data column, or dimension, separately and this has also been looked at previously, usually in relation to nearest neighbour or kNN classifiers [2], for example. The oscillating error technique was a simple rule that introduced the idea of using cellular automata [8][22] as the neural unit, where the small add or subtract decision gave the classifier an added dimension of flexibility. The paper [8] presents a proof that dynamic cellular automata are flexible enough to simulate arbitrary computations, for example, which means algorithms in general. They describe that this has been put in the context of state machines, where classical algorithms were axiomatized and generalised by Gurevich [22].

Deep Learning Networks [10][28], Decision [21] and Regression Trees have made tremendous advances in a lot of areas, but they are still not universal classifiers. Some recent papers [10][28][34] show that they can still have problems with these benchmark datasets. The paper [34] compares classifiers with linear or convex 'hulls' and concludes that the linear hull is prone to overfitting and does not work as well. That is interesting, because while this classifier appears to be linear, it performs well with non-linear data, but has that dimension of flexibility. They note that the convex hull is more constrained and each classifier in this new model is definitely bounded. These established classifiers also tend to require a lot more training and configuring; but the classifier of this paper, probably because of its simplicity, also has clear drawbacks. There is clearly a problem with the design that prevents it being a universal classifier. The new 
classifier may also be of interest with respect to biological models of the human brain, which will be elaborated on later, but papers that note the importance of neuron links in the brain for communication processes include [19][36]. Some of the health datasets have been tested, where a summary of the current state there can be found in [26].

\section{Oscillating ERror Classifier Review}

This section summarises the earlier oscillating error classifier, used to classify data into categories. Essentially, the classifier works off averaged numerical values and does not incrementally update values for each data row. It would create a classifier for each output category and group all of the input data that belonged to the category together. The classifier for that category would then learn to adjust its input, which would be the averaged data row value for the whole data group, to the desired output, which could be set to a value like 1 or 0.5 , because each category was separate. The idea would be simply to have a weight value, to adjust the input with, to move it to the desired output value. The premise for this is the fact that there can only be one weight value for all of the inputs and so learning the averaged value looks reasonable. The oscillating error method added or subtracted the difference between the actual and the desired value from each data column separately. So, for example, the difference could be subtracted from the value in column 1, but added to the value in column 2 and this was a change from a neural network neuron to an automaton. This was repeated until a minimum error or a maximum number of iterations was achieved. The data was also normalised, to be in the range 0 to 1 , so that each adjustment was equal.

This oscillating error process was still not sufficiently accurate however, where lots of data rows would be associated with the wrong classifier. Each classifier would therefore branch to a new level when it contained data rows from more than 1 category, see section 5. Any new level would create a new set of classifiers, one for each associated category and repeat the process in exactly the same way as for the parent level. This new level however would have a less complicated problem to solve, because it would only have to solve a subset of the whole dataset. It has since been discovered that the oscillating error technique is not required for a model that uses a separate classifier for each category and a single adjustment from the averaged value to the desired output value can be used instead. This is the basis for section 4, where the adjustment can still be an addition or subtraction however, and made independently for each column.

When using the classifier then: if it had branches, they were always passed the input data and asked to return their evaluation. If there was only 1 classifier with no branches, it would return its own result, which would be the difference between the weight-adjusted input value and the category value. Therefore, each base classifier would return some category evaluation and error result and the classifier with the smallest error would be selected as the best match. Logically this could lead to a classifier learning a single data row and that might be expected to be $100 \%$ accurate. This does not appear to be the case and overfitting of the data is an obvious problem with it. Overlapping regions in the data values probably lead to some confusion as well.

\section{Map to the Centroid Value}

The first improvement to the classifier is to map directly to the centroid value, to represent the category. Each category is assigned a separate classifier, where the desired category output value can be anything, such as 1 or 0.5 , or in some cases a graded value through all of the categories. The value is not as important as the weight adjustment to it and so the classifier can in fact be simplified from that model by using the category centroid as the desired category output and subsequent weight adjustment. This also helps to add non-linearity, through a curved output line, 
for example. Each new input data row can be compared to the centroid directly and the total absolute difference in the row values taken to be the error. The following example explains how it works: Consider a dataset with 2 categories $\mathrm{A}$ and $\mathrm{B}$. These datasets have the following rows, shown in Figure 1, assigned to them. A new data row has the values '4, 3, 2' and comparing to the centroids gives the error values, also shown in Figure 1. Therefore, the new data row belongs to category A.

\begin{tabular}{c|c}
\hline Category $\boldsymbol{A}$ & Category $\boldsymbol{B}$ \\
Data row: $1,2,1$ & Data row: $5,6,5$ \\
Data row: $1,2,3$ & Data row: $5,6,7$ \\
Data row: $3,2,1$ & Data row: $7,6,5$ \\
Centroid row: $1.66,2,1.66$ & Centroid row: $5.66,6,5.66$ \\
Difference $=2.34+1+0.34=3.68$. & Difference $=1.66+3+3.66=8.32$ \\
\hline
\end{tabular}

Figure 1. Data rows, centroid and error difference with data row ' $4,3,2$ ', for 2 categories.

\section{EXtending The Classifier With BRAnChING}

This section is repeated from the paper [17] and describes the second improvement. The dataset is initially split into groups, where there are $x$ classifiers in the first level, one for each category. Each classifier learns the centroid value for its data subset. The whole dataset is then passed through all of the classifiers again and each produces an error for each data row, as described in Figure 1. After this training phase, there is a list of data rows for each classifier that it has produced the closest match to. Most of the rows would be for the correct category, but some would be matched more closely with other classifiers. The branching extension therefore adds a new level to the classifier, to refine it with respect to the incorrectly classified data rows. The schematic of Figure 2 shows the classification process, where a new layer has been added to classifier A, so that it can correctly re-classify the category A and B sub-groups that belong to it. The second level uses a subset of the whole dataset that is specifically only the data rows assigned to the classifier at the parent level. For the classifier's own category, this is the same as for the first level, but then other categories are represented by new classifiers in the new level.

\subsection{Mathematical Proof}

There is some mathematical justification for why centroid values can be used for the output category values. Because averaged values are used, the problem is to map as closely as possible to these and that is a bit like finding a best fit line. In this case, the best fit line is known and would be the centroid values. Therefore, it makes sense to try to map to this and make it the desired output value as well. The classifier then needs to converge when data rows are incorrectly classified. When training, if the classifier moves to its next layer, it only needs to consider the dataset rows related with its current layer. So, when these are adjusted for incorrect categories, the consideration is for that subset only, which is an easier problem to solve. This is a fairly basic argument for why the classifier should work. 


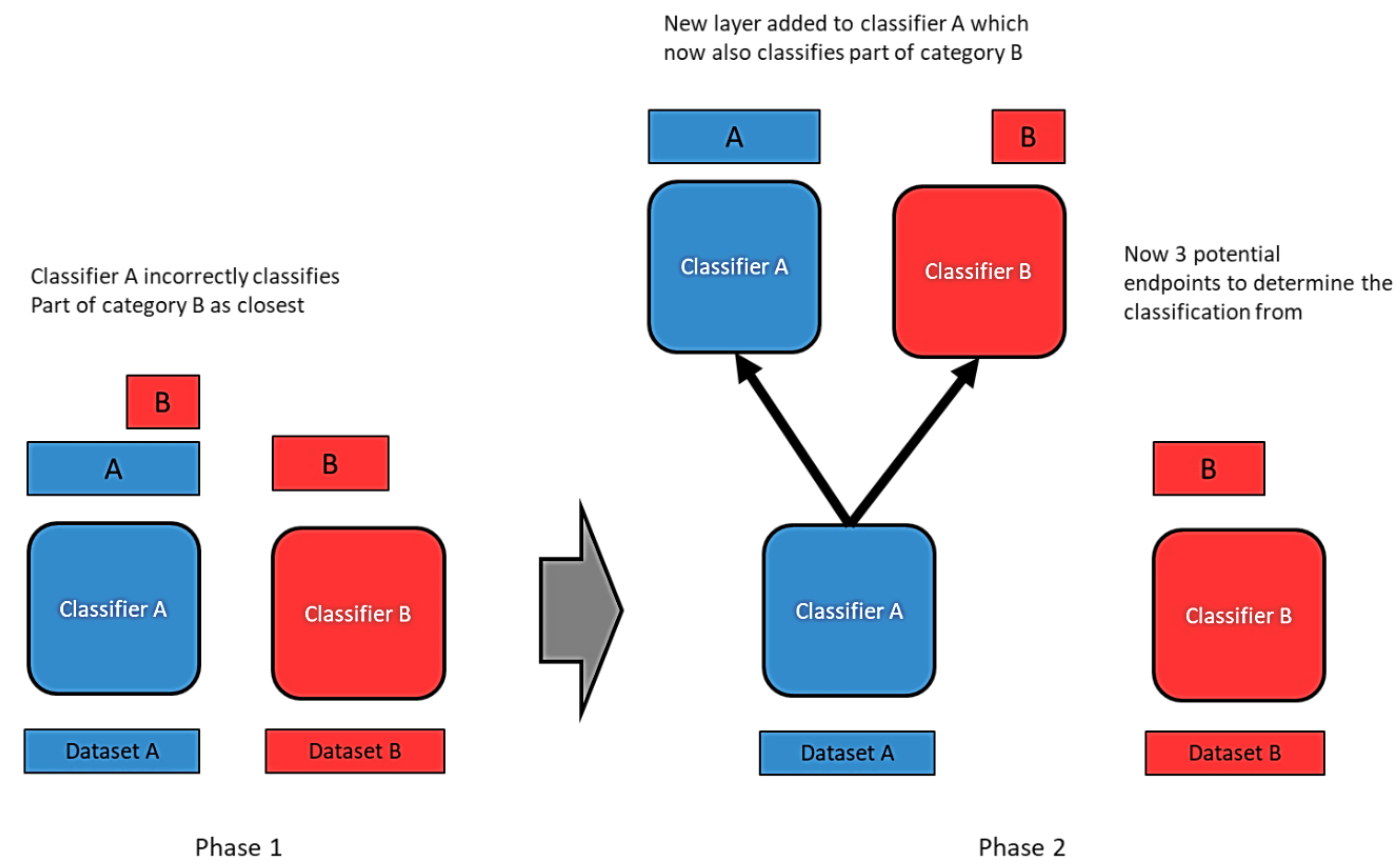

Figure 2. Schematic of the classifier in action. Phase 1 realises that classifier A also classifies part of category B better. Phase 2 then adds a new layer to classifier A, to re-classify this subset only.

\section{EXTending The Classifier with Fixed BandS}

If the classifier deals with averaged values, then it does not try to learn too much outside of that and so one question might be if ranges of input values can be used instead. It would certainly be the case if the data was linearly separable, because the separating line would allow a clear distinction to be determined, but there is still a problem when data from different categories overlap. If trying to separate the input data then, multi-dimensional hypercubes would be the first choice, but this looks like a very difficult problem to solve. Therefore, another option might be to try to separate on each dimension, or feature only and the test results show that this can be quite successful. The process is as follows: The data can be read, one column at a time, as it is organised in the data file. The category that each row belongs to is also retrieved and if there is a change in category, the previous set of values can be placed into a band. The only problem is when categories overlap in a single dimension, which would mean that they have the same value. The band then continues to the next value and category change. For example, consider the following values for a column and related categories, shown in Figure 3.

\begin{tabular}{c|c}
\hline Column Value & Category \\
\hline 0.1 & $\mathrm{~A}$ \\
0.2 & $\mathrm{~A}$ \\
0.3 & $\mathrm{~A}$ \\
0.4 & $\mathrm{~B}$ \\
0.5 & $\mathrm{~B}$ \\
0.5 & $\mathrm{C}$ \\
0.6 & $\mathrm{C}$ \\
\hline
\end{tabular}

Figure 3. Example of Data column values with related categories, placed into bands: Band $1-0.1$ to 0.3 and Band 2 - 0.4 to 0.6 . 
The program would therefore firstly sort the data column values into order. It would then read down the column until there is a change in the category. In this case, the first change is at the value 0.4 . The value range 0.1 to 0.3 all belongs to a single category and so a band can be made from that. Then process continues and the next break would be at the value 0.5 , but there is an overlap with this value as both categories $\mathrm{B}$ and $\mathrm{C}$ use it. Therefore, the process must continue to the end value, when both of those categories are placed in a single value range of 0.4 to 0.6. The process is repeated for each column, to produce a set of bands for the column. It is also important to link the bands from one dimension to the next depending on the exact values in each data row. As an example, if there are 3 columns in a dataset and each column has 5 bands; then if a data row relates to bands 1, 2 and 4, these bands will have links added between them. Then the band 1 relating to column or dimension 1 can only move to band 2 in the next dimension, and so on.

Figure 4 shows the bands and links created for the Iris Plants dataset [11]. When presented with a new data row to evaluate, the procedure traces through the band links, to check if any represent a single category only. If that is the case, then the fixed band ranges can be used to classify the input data directly. Because of the overlap however, there are lots of cases where a band represents more than 1 category. It would be interesting to train the classifiers for those cases only, but for a first test, the classifier system with branching was also trained and used if the bands did not return a result. The bands can help with this a bit however, because if they can classify any data rows directly, those rows do not need to be considered by the classifiers and so this was also checked for and those data rows removed from the training dataset. The bands may therefore be able to make a contribution to the classification process. For example, Figure 4 shows bands, where category 1 is linearly separable and can be identified completely from using the band ranges. Categories 2 and 3 would need a classifier to be separated. Also, for category 1 , the classification is clear from column 3 and so column 4 is probably not required. In effect, column 3 provides a unique feature for category 1 .

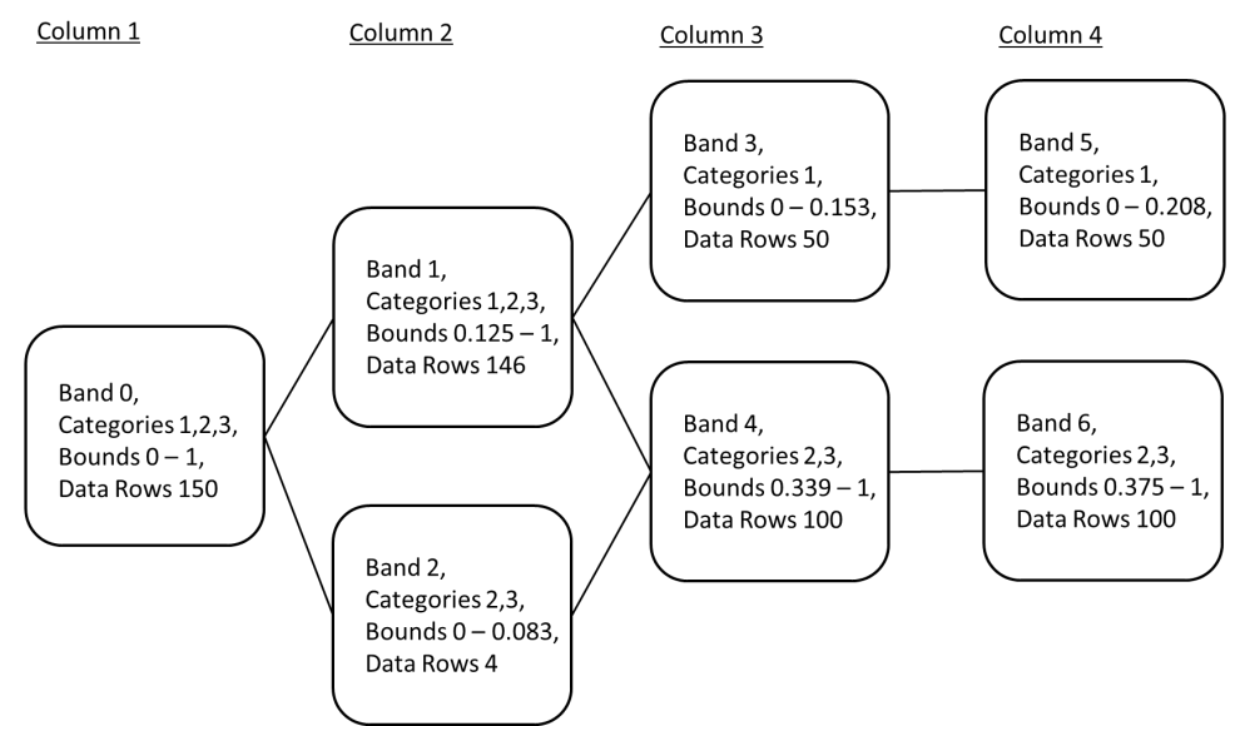

Figure 4. Bands created for the Iris Plants dataset [11].

\section{TeSt Results}

This paper repeats the set of tests carried out in the earlier paper [18], to verify the classifier accuracy and also includes some new datasets. A test program has been written in the C\# .Net language. It can read a file of data, normalise it, generate the classifier from it and measure how 
many categories it subsequently evaluates correctly. Two types of result were measured. The first was an average error for each row in the dataset, after the classifier was trained. This was calculated as the average difference between the input vector and the output centroid vector. This is only a minor result and the second more important measurement was how many categories were correctly classified. For these tests, the error 'margin' used in the original tests was not required, where the errors of each output classifier could be compared directly and the smallest one selected.

\subsection{Benchmark Datasets with Train Versions Only}

The classifier was first tested on several datasets from the UCI Machine Learning Repository [39]. These included the Wine Recognition [12], Iris Plants [11] and the Zoo [41] databases. Wine Recognition and Iris Plants have 3 categories, while the Zoo database has 7. These do not have a separate training dataset and are benchmark tests for classifiers. In fact, the classifier trains in a single step and so it does not require a stopping criterion, but it could be prone to overtraining if layers are continually added. For the Wine dataset, the UCI [39] web page states that the classes are separable, but only RDA [14] has achieved 100\% correct classification. Other datasets included the Abalone shellfish dataset [1], the Hayes-Roth concept learning dataset [24] and the BUPA Liver dataset [32]. Then the Cleveland Heart Disease [9] and the Breast Cancer [25] datasets were also tested. While the Heart Disease dataset was originally tested for presence (cats 1-4) or absence (cat 0 ), this test matched with the output category exactly, resulting in 5 output categories.

Another web site [5] lists other datasets, where tested here was the Sonar [16][40] and Wheat Seeds [6] datasets, with previous benchmark results of $100 \%$ and $92 \%$ respectively. The Car [3][35] and Wine Quality [7] datasets were also tested. As shown in Table 1, the new classifier produces a remarkable result of $100 \%$ accuracy over all of these datasets. The Wine dataset also produced a $100 \%$ accuracy over a 45 -row test set and a 133-row train set, for example. The column 'Other Best \%' lists a result found by other researchers, but may be slightly out of date due to the recent advances. The final column indicates if feature bands could be used and if they were better. For these tests, using bands was not particularly useful and improved the result in only the Hayes-Roth case.

Table 1. Classifier Test results. Average output error and number of correct classifications. All datasets points normalised to be in the range 0 to 1 .

\begin{tabular}{|c|c|c|c|c|c|}
\hline Dataset & Av Error & Number Correct & \% Correct & Other Best \% & Use Bands \\
\hline Wine & 0.1 & 178 from 178 & 100 & 100 & Either \\
\hline Iris & 0.07 & 150 from 150 & 100 & 97 & Either \\
\hline Zoo & 0.1 & 101 from 101 & 100 & 94.5 & Either \\
\hline Abalone & 0.01 & 4177 from 4177 & 100 & 73 & Either \\
\hline Hayes-Roth & 0 & 132 from 132 & 100 & 50 & Yes \\
\hline Liver & 0.08 & 345 from 345 & 100 & 74 & Either \\
\hline Cleveland & 0.15 & 303 from 303 & 100 & 77 & Either \\
\hline Breast & 0.08 & 569 from 569 & 100 & 98.5 & Either \\
\hline Sonar & 0.15 & 208 from 208 & 100 & 100 & Either \\
\hline Wheat & 0.09 & 210 from 210 & 100 & 92 & Either \\
\hline Car & 0.28 & 1728 from 1728 & 100 & 97 & Either \\
\hline Wine Quality & 0.06 & 1599 from 1599 & 100 & 89 & Either \\
\hline
\end{tabular}




\subsection{Separate Train and Test Datasets}

There is an important question about generalisation properties when averaged values and bands are used, and the fact that batch data rows can be reduced to a size of 1 . A slightly better test would therefore be to have different train and test datasets and this section gives the results for those tests, shown in Table 2. The training time would be instantaneous for something like the Iris Plants dataset, but for the Letter Recognition dataset in this section, it took much longer. But the system is very low on resource usage and the process is completely deterministic, meaning that it should give exactly the same result each time. There is no fine tuning either.

Table 2. Classifier Test results. The same criteria as for Table 1, but a separate test dataset to the train dataset.

\begin{tabular}{|c|c|c|c|c|c|}
\hline Dataset & Av Error & Number Correct & \% Correct & Other Best \% & Use Bands \\
\hline UM & 0.17 & 144 from 145 & 99.9 & 98 & No \\
\hline Bank & 0.15 & 100 from 100 & 100 & 61 & No \\
\hline SPECT & 0.14 & 187 from 187 & 100 & 84 & No \\
\hline Letters & 0.07 & 3623 from 4000 & 90 & 82 & No \\
\hline Monks-1 & 0.35 & 432 from 432 & 100 & 100 & Either \\
\hline Solar & 0.05 & 1017 from 1066 & 95 & 84 & Yes \\
\hline Diabetes & 0.12 & 368 from 368 & 100 & 77 & No \\
\hline Ionosphere & 0.15 & 150 from 150 & 100 & 96 & Either \\
\hline
\end{tabular}

This other set of test results, using separate train and test datasets, was as follows: The first set of datasets were User Modelling [27], Bank Notes [33], SPECT images heart classification [30], Letter recognition [13], the first Monks dataset [38] and Solar flares [4][31]. Then two other datasets were artificially split into train and test sets. The Pima Indians Diabetes dataset [29] was split into a train set of 400 rows and a test set of 368 rows. The Ionosphere dataset [37] was divided into a train set of 201 rows and a test set of 150 rows. Both of these performed equally well on the whole dataset. It can be seen that for these tests the classifiers preferred not to use fixed bands and so the generalising properties of the bands is less than for the classifier itself.

\subsection{Test Conclusions}

The new version that includes bands is certainly worth considering, even if the earlier version can produce better results in most cases. Both versions are very easy to train and use. The size of the whole structure is quite large, but it is also very simple and the same each time. For example, for the Abalone dataset, 2700 classifiers were created and the Letters dataset produced 12600 classifiers, where most of that would be branching to next levels. In real time, it might then have to test the input across that number of classifiers as well, which is quite a lot for a relatively small dataset. While using bands should reduce the number of data rows that a classifier needs to learn, it typically resulted in even more classifiers, which is a surprise and so there may be a coherence factor as the data gets split. They were also shown not to be as useful on previously unseen data and so their generalisation properties are not as good. However, for a couple of datasets, they provided better results. While each classifier solves only a small part of the larger problem, it is not the case that each classifier has been given a few rows of data to classify. The system has generated the classifiers and row sets for itself. It should also be possible to update the system dynamically. 


\subsubsection{Dataset Normalisation}

The results in Table 1 are very impressive, but maybe not the whole story. The Wine Quality dataset [7] illustrates another point. For one test, it was divided into a train set of 1100 data rows and a separate test set of 499 rows. Normalising the data was then done in one of two ways. The first way would be to normalise over each dataset separately. This would potentially create different minimum and maximum values for each dataset. The other way would be to normalise over both datasets together and use the same minimum and maximum value to normalise either with. This could potentially change the result of the classification for a previously unseen dataset. In the case of the wine quality data, if the train and test sets were normalised separately, the test set would be recognised at only $86.5 \%$ accuracy (431 correct from 499). If the datasets were normalised together, then the test set was recognised at 90\% accuracy (451 correct from 499), which is on a par with the other selected best value. This is therefore another factor to consider and could lead to better results if the correct option is chosen.

\section{Conclusions}

This paper has extended the work reported in [17][18], with two improvements. These are to use the centroid as the matching criterion for the output category and to branch the classifier when some data rows are incorrectly classified. As the structure is now a tree, the classifier can be called Category Trees. The idea of fixed bands is also introduced, where these bands can be used to classify some input data directly, simply by using value ranges. The bands and the classifiers are currently trained separately and are not linked-up, but the bands can remove some data rows for the training of the classifiers. The test results show that it can out-perform a lot of other classifiers and is probably easier to use. The exact nature of the classifier is now a lot clearer. It simply maps to the averaged values through a linear adjustment, but non-linearities can benefit from the distributed and unrelated nature of the weight-adjustment values. These non-linearities however, can be built-up systematically and holistically, which explains why it can classify something like the Abalone shellfish dataset [1] better. It is not helped by other methods, for overfitting for example, but the results are such that it would take something unusual to improve it further.

\subsection{Biological Discussion}

Comparing the classifier with something like PCA, feature selection and biological systems can lead to some logical conclusions. If a data object has a unique feature (data column) then a data band can use that to classify it directly. Maybe in effect, it could be passed down a unique channel relating to that band. If the data object does not have a unique feature, then the current system takes all of the features together and compares that with an averaged value of the features present in each category. The data object is then allocated the category that it is a closest match to. The comparison with neurons and links between them is clear. There is biological evidence that the links between neurons play an important role in the actual signal interpretation and understanding in the human brain [36]. The bands would therefore be an analogy to the neuron links. If the signal fits inside of the band boundaries, it can be classified as whatever the band represents without a neuron interpreting it further. If there is any discrepancy, then a classifier is required to sort that out and so this is analogous to a neuron being created to process a more mixed signal. If the neuron behaves like a filter, then the process might be to convert the mixed signal back into more singular parts again. 
International Journal of Artificial Intelligence \& Applications (IJAIA), Vol.12, No.6, November 2021

\section{REFERENCES}

[1] Asim, A., Li, Y., Xie, Y. and Zhu, Y. (2002). Data Mining for Abalone, Computer Science 4TF3 Project, Supervised by Dr. Jiming Peng, Department of Computing and Software, McMaster University, Hamilton, Ontario.

[2] Bay, S.D. (1999). Nearest Neighbor Classification from Multiple Feature Subsets, Intelligent data analysis, Vol. 3, No. 3, pp. 191-209.

[3] Bohanec, M. and V. Rajkovic, V. (1988). Knowledge acquisition and explanation for multi-attribute decision making. In 8th Intl Workshop on Expert Systems and their Applications, Avignon, France, pp. 59-78.

[4] Bradshaw, G. Donor of the Solar Flares dataset, Gary Bradshaw <gbradshaw@ @lipr.colorado.EDU>. (last accessed (18/11/19).

[5] Brownlee, J. (2019). 10 Standard Datasets for Practicing Applied Machine Learning, https://machinelearningmastery.com/standard-machine-learning-datasets/.

[6] Charytanowicz, M., Niewczas, J., Kulczycki, P., Kowalski, P.A., Lukasik, S. and Zak, S. (2010). A Complete Gradient Clustering Algorithm for Features Analysis of X-ray Images, in: Information Technologies in Biomedicine, Ewa Pietka, Jacek Kawa (eds.), Springer-Verlag, Berlin-Heidelberg, pp. $15-24$.

[7] Cortez, P., Cerdeira, A., Almeida, F., Matos T. and Reis, J. (2009). Modeling wine preferences by data mining from physicochemical properties. In Decision Support Systems, Elsevier, Vol. 47, No. 4, pp. 547-553.

[8] Dershowitz, N. and Falkovich, E. (2015). Cellular Automata are Generic, U. Dal Lago and R. Harmer (Eds.): Developments in Computational Models 2014 (DCM 2014). EPTCS 179, pp. 17-32, doi:10.4204/EPTCS.179.2.

[9] Detrano, R., Janosi, A., Steinbrunn, W., Pfisterer, M., Schmid, J., Sandhu, S., Guppy, K., Lee, S. and Froelicher, V. (1989). International application of a new probability algorithm for the diagnosis of coronary artery disease. American Journal of Cardiology, Vol. 64, pp. 304 - 310.

[10] Dong, M., Yao, L., Wang, X., Benatallah, B. and Zhang, S. (2018). GrCAN: Gradient Boost Convolutional Autoencoder with Neural Decision Forest. arXiv preprint arXiv:1806.08079.

[11] Fisher, R.A. (1936). The use of multiple measurements in taxonomic problems, Annual Eugenics, 7, Part II, pp. 179-188, also in 'Contributions to Mathematical Statistics' (John Wiley, NY, 1950).

[12] Forina, M. et al. (1991). PARVUS - An Extendible Package for Data Exploration, Classification and Correlation. Institute of Pharmaceutical and Food Analysis and Technologies, Via Brigata Salerno, 16147 Genoa, Italy.

[13] Frey, P.W. and Slate, D.J. (1991). Letter recognition using Holland-style adaptive classifiers, Machine learning, Vol. 6, No. 2, pp. 161-182.

[14] Friedman, J.H. (1989). Regularized Discriminant Analysis, Journal of the American Statistical Association, Vol. 84, No. 405, pp. 165-175.

[15] Gallant, S.I. (1990). Perceptron-Based Learning Algorithms, IEEE Transactions on Neural Networks, Vol. 1, No. 2.

[16] Gorman, R.P., and Sejnowski, T.J. (1988). Analysis of Hidden Units in a Layered Network Trained to Classify Sonar Targets, Neural Networks, Vol. 1, pp. 75 - 89.

[17] Greer, K. (2017). An Improved Oscillating-Error Classifier with Branching, WSEAS Transactions on Computer Research, Vol. 6, pp. 49 - 54. E-ISSN: 2415-1521.

[18] Greer, K. (2017). A New Oscillating-Error Technique for Classifiers, Cogent Engineering, Taylor and Francis Online, https://doi.org/10.1080/23311916.2017.1293480.

[19] Greer, K. (2015). New Ideas for Brain Modelling, BRAIN. Broad Research in Artificial Intelligence and Neuroscience, Volume 6, Issues 3-4, pp 26 - 46, December 2015, ISSN 2067-3957 (online), ISSN 2068 - 0473 (print).

[20] Greer, K. (2013). Artificial Neuron Modelling Based on Wave Shape, BRAIN. Broad Research in Artificial Intelligence and Neuroscience, Vol. 4, Nos. 1-4, pp. 20- 25, ISSN 2067-3957 (online), ISSN 2068 - 0473 (print).

[21] Gupta, B., Rawat, A., Jain, A., Arora, A. and Dhami, N. (2017). Analysis of various decision tree algorithms for classification in data mining. International Journal of Computer Applications, Vol. 163 , No. 8, pp. 15 - 19. 
[22] Gurevich, Y. (2000). Sequential Abstract State Machines Capture Sequential Algorithms, ACM Transactions on Computational Logic Vol. 1, No. 1, pp. 77 - 111, doi:10.1145/343369.343384. Available at http://research.microsoft.com/ gurevich/ opera/141.pdf.

[23] Hagan, M.T. and Menhaj, M.B. (1994). Training Feedforward Networks with the Marquardt Algorithm, IEEE Transactions on Neural Networks, Vol. 5, No. 6, pp. 989-993.

[24] Hayes-Roth, B. and Hayes-Roth, F. (1977). Concept Learning and the Recognition and Classification of Exemplars, Journal of Verbal Learning and Verbal Behavior, Vol. 16, No. 3, pp. 321-338.

[25] Hu, Y. C. (2007). Fuzzy integral-based perceptron for two-class pattern classification problems. Information Sciences, Vol. 177, No. 7, pp. 1673 - 1686.

[26] Jha, S.K., Pan, Z., Elahi, E. and Patel, N. (2019). A comprehensive search for expert classification methods in disease diagnosis and prediction. Expert Systems, Vol. 36, No. 1, p.e12343.

[27] Kahraman, H.T., Sagiroglu, S. and Colak, I. (2013). The development of intuitive knowledge classifier and the modeling of domain dependent data, Knowledge-Based Systems, Vol. 37, pp. $283-$ 295.

[28] Katuwal, R., Suganthan, P.N. (2019). Stacked Autoencoder Based Deep Random Vector Functional Link Neural Network for Classification, accepted: Applied Soft Computing (https: // doi. org/ 10. 1016/ j. asoc. 2019. 105854).

[29] Keller, F., Muller, E. and Bohm, K. (2012). HiCS: High-contrast subspaces for density-based outlier ranking, IEEE 28th International Conference on Data Engineering (ICDE), pp. 1037 - 1048.

[30] Kurgan, L.A., Cios, K.J., Tadeusiewicz, R., Ogiela, M. and Goodenday, L.S. (2001). Knowledge Discovery Approach to Automated Cardiac SPECT Diagnosis, Artificial Intelligence in Medicine, Vol. 23, No. 2, pp. 149-169.

[31] Li, J., Dong, G. and Ramamohanarao, K. (2000). Instance-based classification by emerging patterns. In European Conference on Principles of Data Mining and Knowledge Discovery, pp. 191 - 200, Springer, Berlin, Heidelberg.

[32] Liver dataset (2016). https://archive.ics.uci.edu/ml/datasets/Liver+Disorders.

[33] Lohweg, V., Dörksen, H., Hoffmann, J. L., Hildebrand, R., Gillich, E., Schaede, J., and Hofmann, J. (2013). Banknote authentication with mobile devices. In IS\&T/SPIE Electronic Imaging (pp. 866507-866507). International Society for Optics and Photonics.

[34] Nguyen, T., Ye, N. and Bartlett, P.L. (2019). Learning Near-optimal Convex Combinations of Basis Models with Generalization Guarantees. arXiv preprint arXiv:1910.03742.

[35] Oza, N.C. and Russell, S. (2001). Experimental comparisons of online and batch versions of bagging and boosting. In Proceedings of the seventh ACM SIGKDD international conference on Knowledge discovery and data mining, pp. 359-364.

[36] Shira Sardi, S., Vardi, R., Sheinin, A., Goldental, A. and Kanter, I. (2017). New Types of Experiments Reveal that a Neuron Functions as Multiple Independent Threshold Units, Nature Scientific Reports, 7:18036, DOI:10.1038/s41598-017-18363-1.

[37] Sigillito, V.G, Wing, S.P, Hutton, L.V. and Baker, K.B. (1989). Classification of radar returns from the ionosphere using neural networks, Johns Hopkins APL Tech. Dig, Vol. 10, pp. 262 - 266.

[38] Thrun, S.B., Bala, J., Bloedorn, E., Bratko, I., Cestnik, B., Cheng, J.,nDe Jong, K., Dzeroski, S., Fahlman, S.E., Fisher, D., Hamann, R., Kaufman, K., Keller, S., Kononenko, I., Kreuziger, J., Michalski, R.S., Mitchell, T., Pachowicz, P., Reich Y., Vafaie, H., Van de Welde, W., Wenzel, W., Wnek, J. and Zhang, J. (1991). The Monk's Problems - A Performance Comparison of Different Learning Algorithms, has been published as Technical Report CS-CMU-91-197, Carnegie Mellon University in Dec. 1991.

[39] UCI Machine Learning Repository (2019). http://archive.ics.uci.edu/ml/.

[40] Zhou, Z.H. and Jiang, Y. (2004). NeC4. 5: neural ensemble based C4. 5. IEEE Transactions on Knowledge and Data Engineering, Vol. 16, No. 6, pp.770 - 773.

[41] Zoo database (2016). https://archive.ics.uci.edu/ml/datasets/Zoo. 
International Journal of Artificial Intelligence \& Applications (IJAIA), Vol.12, No.6, November 2021

\section{AuTHORS}

Kieran Greer is a freelance software engineer and researcher. He has worked at the two Universities in Northern Ireland and also runs his own Software R\&D company, Distributed Computing Systems (.co.uk). He specialises in the areas of Artificial Intelligence and Information Systems. He has developed new Artificial Intelligence models, and software products include 'licas' and 'Textflo'.

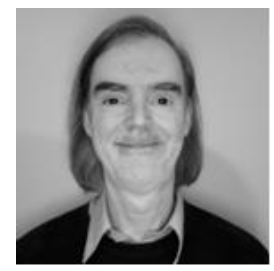

